\title{
CONTINUUM LIMITS OF PARTICLES INTERACTING VIA DIFFUSION
}

\author{
NICHOLAS D. ALIKAKOS, GIORGIO FUSCO, AND GEORGIA KARALI
}

Received 5 August 2003

We consider a two-phase system mainly in three dimensions and we examine the coarsening of the spatial distribution, driven by the reduction of interface energy and limited by diffusion as described by the quasistatic Stefan free boundary problem. Under the appropriate scaling we pass rigorously to the limit by taking into account the motion of the centers and the deformation of the spherical shape. We distinguish between two different cases and we derive the classical mean-field model and another continuum limit corresponding to critical density which can be related to a continuity equation obtained recently by Niethammer and Otto. So, the theory of Lifshitz, Slyozov, and Wagner is improved by taking into account the geometry of the spatial distribution.

\section{Introduction}

Ostwald ripening is a very general phenomenon occurring in liquids, solids, and on solid surfaces. Basically, it takes place in the late stages of first-order phase transitions, when larger droplets, grains, crystallites, or islands grow at the expense of smaller ones via evaporation, diffusion, and condensation. In general, the kinetics of a first-order phase transition are characterized by a first stage where small droplets of a new phase are created out of the old phase, for example, solid formation in an undercooled liquid. The first stage, called nucleation, yields a large number of small particles. During the next stage, the nuclei grow rapidly at the expense of the old phase. When the phase regions are formed, the mass of the new phase is close to equilibrium and the amount of undercooling is small, but large surface area is present. In the next stage, the configuration of phase regions is coarsened, and the geometric shape of the phase regions becomes simpler and simpler, eventually tending to regions of minimum surface area with given volume. The driving force of this process comes from the need to decrease interfacial energy with increasing the average size. There have been considerable efforts in finding a theory which describes Ostwald ripening, and the Mullins-Sekerka model is a prominent candidate. In the present work we focus on this model. The Mullins-Sekerka model is a nonlocal evolution law in which the normal velocity of a propagating interface depends on the 
jump across the interface of the normal derivative of a function which is harmonic on either side and which equals the mean curvature on the propagating interface. The model was first introduced by Mullins and Sekerka [6] to study solidification and liquidation of materials and it has the following form

$$
\begin{gathered}
-\Delta u=0, \quad \text { off } \Gamma(t), \text { in } \Omega \subset \mathbb{R}^{3}, \\
u=H, \quad \text { on } \Gamma(t), \\
\frac{\partial u}{\partial V}=0, \quad \text { on } \partial \Omega, \\
V=\left[\left[\frac{\partial u}{\partial n}\right]\right], \quad \text { on } \Gamma(t),
\end{gathered}
$$

where $\Omega$ is a bounded, smooth, simply connected domain; $u$ is the chemical potential, $H$ is the mean curvature of $\Gamma(t) ; V$ is the normal velocity taken positive for a shrinking sphere; $[[\partial u / \partial n]]=\left(\partial u_{e} / \partial n_{e}\right)+\left(\partial u_{i} / \partial n_{i}\right)$ is the jump of the derivative of $u$ in the normal direction to $\Gamma$ where $u_{e}, u_{i}$ are the restrictions of $u$ on the exterior $\Omega_{e}$ and the interior $\Omega_{i}$ of $\Gamma(t)$ in $\Omega$ and $n_{e}, n_{i}$ are the unit exterior normal to $\Omega_{e}, \Omega_{i}$; and $\Gamma=\bigcup_{i=1}^{N} \Gamma_{i}$ is the union of the boundaries of the $N$ particles. The Mullins-Sekerka model arises as a singular limit for the Cahn-Hilliard equation, see $[1,10]$. Solutions to the Mullins-Sekerka model evolve in such a way that the volume of the region enclosed by $\Gamma(t)$ is preserved, while the area of $\Gamma(t)$ shrinks. This is a consequence of the following simple computations provided that the interfaces exist and are smooth. Let $A(t)$ denote the area of $\Gamma(t)$ and $\operatorname{Vol}(t)$ the volume of the enclosed region $\Omega_{i}(t)$. Then one can compute

$$
\begin{aligned}
\frac{d}{d t} \operatorname{Vol}(t) & =-\int_{\Gamma} V=\int_{\Gamma}\left[\frac{\partial u}{\partial n}\right]=\int_{\Omega \backslash \Gamma} \Delta u=0, \\
\frac{d}{d t} A(t) & =-\int_{\Gamma} H V=-\int_{\Gamma} u\left[\frac{\partial u}{\partial n}\right]=-\int_{\Omega}|\nabla u|^{2} \leq 0 .
\end{aligned}
$$

A single sphere or multiple spheres of the same radius are equilibria for the evolution. In $[2,4]$ an initial configuration of a finite number $N$ of almost spherical particles was considered. It was shown that to each particle we can associate a center $\xi \in \mathbb{R}^{3}$, a radius $R \in \mathbb{R}^{+}$, and a shape function $r(\cdot) \in C^{3+\alpha}\left(S^{2}\right)$ such that

$$
\Gamma_{i}(t)=\left\{x / x=\xi_{i}+\epsilon R_{i}\left(1+\epsilon r_{i}(u)\right) u, u \in S^{2}\right\}
$$

with $r_{i}$ satisfying the orthogonality conditions

$$
\begin{gathered}
\int_{S^{2}} r_{i}(u) d u=0, \quad i=1, \ldots, N, \\
\int_{S^{2}} r_{i}(u)\left\langle u, e_{j}\right\rangle d u=0, \quad j=1,2,3,
\end{gathered}
$$

where $\langle\cdot, \cdot\rangle$ is the Euclidean inner product, $e_{j} \in \mathbb{R}^{3}, j=1,2,3$, is the standard unit vector, and $S^{2}$ is the unit sphere. We derive equations for the radii and the centers which have the 
following expressions:

$$
\begin{aligned}
& \frac{d R_{i}}{d \tau}=\frac{1}{\epsilon^{3} R_{i}}\left\{\left(\frac{1}{\bar{R}}-\frac{1}{R_{i}}\right)\right. \\
& +\epsilon\left[\frac{1}{N \bar{R}} \sum_{\substack{k, h \\
k \neq h}} \frac{R_{h}}{\left|\xi_{h}-\xi_{k}\right|}\left(\frac{R_{k}}{\bar{R}}-1\right)-\sum_{j \neq i} \frac{1}{\left|\xi_{j}-\xi_{i}\right|}\left(\frac{R_{j}}{\bar{R}}-1\right)\right. \\
& \left.\left.+\frac{1}{N} \sum_{k, h} 4 \pi \frac{R_{k}}{\bar{R}} \gamma\left(\xi_{k}, \xi_{h}\right)\left(\frac{R_{h}}{\bar{R}}-1\right)-\sum_{h} \gamma\left(\xi_{i}, \xi_{h}\right) 4 \pi\left(\frac{R_{h}}{\bar{R}}-1\right)+g_{i}\right]\right\}, \\
& \frac{d \xi_{i}}{d \tau}=3 \sum_{h \neq i} \frac{\xi_{h}-\xi_{i}}{\left|\xi_{h}-\xi_{i}\right|^{3}}\left(1-\frac{R_{h}}{\bar{R}}\right)+3 \sum_{h \neq i} \frac{\partial \gamma}{\partial \xi_{h}}\left(1-\frac{R_{h}}{\bar{R}}\right)+\phi_{i}
\end{aligned}
$$

with

$$
\bar{R}=\frac{1}{N} \sum_{i=1}^{N} R_{i},
$$

where $g_{i}$ and $\phi_{i}$ are precisely estimated higher-order terms, and $\gamma$ is the smooth part of a Green's function capturing the effect of the boundary. Equations (1.5) without $\gamma$ were derived formally by Weins and Cahn [12] as a correction to the classical LifshitzSlyozov-Wagner (LSW) kinetic law that is given by the principal term in (1.5). Ignoring $g_{i}$ and $\phi_{i},(1.5)$ and (1.6) form a closed system for the radii and the centers. The purpose of the present paper is the rigorous continuum limit of (1.5), (1.6) under two different scalings and under no extra assumptions. The resulting continuity equations determine the evolution of the distribution function $n(R, \xi, t)$ which gives the particle density of size $R$, at the location $\xi$, and at time $t$. They have the following form:

$$
\frac{\partial n}{\partial t}+\frac{\partial}{\partial R}\left(\frac{d R}{d t} n(R, \xi, t)\right)=0
$$

with subcritical density

$$
\frac{d R}{d t}=\frac{1}{R}\left(\frac{1}{R}-\frac{1}{R}\right), \quad \frac{d \xi}{d t}=0,
$$

and critical density

$$
\begin{aligned}
& \frac{d R}{d t}=\frac{1}{R}\left\{\left(\frac{1}{\bar{R}}-\frac{1}{R}\right)+K(t)-\int_{0}^{\infty} \int_{\Omega}\left(\frac{1}{|\xi-\eta|}+4 \pi \gamma(\xi, \eta)\right)\left(\frac{R}{\bar{R}}-1\right) n(R, \xi, t) d \xi d R\right\} \\
& \frac{d \xi}{d t}=0
\end{aligned}
$$


where $K(t)$ is determined by the conservation of volume. The subcritical case corresponds to the classical LSW model. The critical versus the noncritical can be understood also as follows. Consider

$$
\begin{gathered}
\Delta u=0, \quad \text { on } \mathbb{R}^{3} \backslash \bigcup_{k} B\left(\xi_{k}, R_{k}\right), \\
u=\frac{1}{R_{i}}, \quad \text { on } \partial B_{i}, i=1, \ldots, N,
\end{gathered}
$$

and examine the validity of the approximate solution $u(x)=\sum_{k=1}^{N}\left(1 /\left|\xi_{k}-x\right|\right)$. (In a very recent preprint, Niethammer and Velázquez [9] have obtained a remarkable estimate for the effective potential of a single particle in the supercritical case by taking into account the screening effect of the surrounding particles. In our setting of bounded domain and hence bounded volume, the limit in the supercritical case does not exist. In the infinite volume case, it does as has been shown in [9]). Clearly $u$ satisfies the equation. Evaluating it on $\partial B_{i}$, we see that the approximate solution $u(x)$ is estimated off by

$$
\left.\sum_{k \neq i}^{N} \frac{1}{\left|\xi_{k}-x\right|}\right|_{x \in \partial B_{i}} \simeq \sum_{k \neq i}^{N} \frac{1}{\left|\xi_{k}-\xi_{i}\right|} .
$$

Thus $\beta=2 / 3$ represents the boundary of the range of validity of this approximation. Notice that the volume fraction is $\epsilon^{3 \beta}$ (cf. Section 2) and tends always to zero as $\epsilon \rightarrow 0$. So, the measure of the $\xi$ 's in the limit is always zero. However, if $\beta=2 / 3$, the electrostatic capacity is nonzero. This was noted in [7] and can be deduced from the remark above. It was noticed in [2], under the appropriate scaling (cf. Section 2), that the estimate $\sum_{i \neq j}\left(1 /\left|\xi_{i}-\xi_{j}\right|\right)=O\left(\epsilon^{3 \beta-2}\right)$ holds. Thus $\beta>2 / 3$ guarantees that this sum goes to zero as $\epsilon \rightarrow 0$. This coincides with the condition that the relative capacity tends to zero as $\epsilon \rightarrow 0$. The case $\beta=2 / 3$ is critical and leads to a qualitatively different continuum limit.

The subcritical case was first derived rigorously by Niethammer [7] for an averaged version of (1.1) that allows the restriction of the evolution to the class of spherical particles. Also [7] assumes immobile centers arranged on a regular periodic lattice on $\mathbb{R}^{3}$. For the rigorous justification of these hypotheses, see $[3,11]$. The critical case represents a significant correction to the classical LSW model which takes into account the geometry of the distribution in space. A closely related continuity equation to (1.10) was derived recently by Niethammer and Otto [8] where the uniformity in space in particular makes a difference in the form of the equations. The structure of the paper is as follows. In Section 2, we introduce our scaling and we present the three fundamental estimates which will allow us to pass to the limit: the monopole approximation, the boundary estimate, and the dipole estimate concerning the estimation of the centers. In Section 3, utilizing an appropriate representation of the measure, we obtain two distinct limits corresponding to the subcritical and critical density. In passing to the limit in Section 3, we follow closely $[7,8]$. 


\section{The basic estimates}

We consider three characteristic length scales in the problem: the size of the particle, the distortion from the spherical shape, and the distance between particles. There are two equivalent normalizations that we can adopt

(i) the size of particle $=O(1), \Lambda=$ size of the domain $=O(1 / \epsilon)$, and the distance between particles $=O\left(1 / \epsilon^{\beta}\right), 0<\beta \leq 1$, where size/distance $=\epsilon^{\beta}$;

(ii) the size of particle $=O(\epsilon), \Lambda=$ size of the domain $=O(1)$, and the distance between particles $=O\left(\epsilon^{\eta}\right), \eta<1$, where size/distance $=\epsilon^{1-\eta}$.

The relation between $\eta$ and $\beta$ is $\beta=1-\eta$. For $\beta=1, \eta=0$, we have the corresponding finite case. We find it convenient to work with scaling (ii). Under the first scaling, the extinction time is $O(1)$ while under the second scaling, the extinction of the particle happens at $O\left(\epsilon^{3}\right)$. Although we adopt the second scaling where the size of the particle is $O(\epsilon)$, we rescale time by setting $\tau=\epsilon^{3} t$. In this new setting, the extinction time is of the order of $T=O(1)$.

In what follows we denote by $N$ the number of active particles, particles for which $R_{i}^{\epsilon}(t)>0$. We denote by $N_{\Omega}$ the initial number of particles where according to our scaling

$$
\begin{aligned}
N_{\Omega} & =\frac{(\text { domain })^{3}}{(\text { distance between particles })^{3}}=\frac{1}{\epsilon^{3 \eta}}, \\
\text { volume fraction } & =\frac{(\text { number of particles })(\text { radius })^{3}}{(\text { domain })^{3}}=\frac{\epsilon^{-3 \eta} \epsilon^{3}}{1}=\epsilon^{3(1-\eta)} .
\end{aligned}
$$

The $R, \xi$ equations after rescaling time take the form

$$
\begin{aligned}
& \frac{d R_{i}}{d t}=\frac{1}{R_{i}}\left\{\left(\frac{1}{\bar{R}}-\frac{1}{R_{i}}\right)+\epsilon\right. \frac{1}{N \bar{R}} \sum_{\substack{k, h \\
k \neq h}} \frac{R_{h}}{\left|\xi_{h}-\xi_{k}\right|}\left(\frac{R_{k}}{\bar{R}}-1\right)-\sum_{i \neq j} \frac{1}{\left|\xi_{i}-\xi_{j}\right|}\left(\frac{R_{j}}{\bar{R}}-1\right) \\
&\left.\left.+\frac{1}{N} \sum_{k, h} 4 \pi \frac{R_{k}}{\bar{R}} \gamma\left(\xi_{k}, \xi_{h}\right)\left(\frac{R_{h}}{\bar{R}}-1\right)-\sum_{h} \gamma\left(\xi_{i}, \xi_{h}\right) 4 \pi\left(\frac{R_{h}}{\bar{R}}-1\right)\right]\right\} \\
&+ O\left(\epsilon^{2(1-\eta)}\right), \\
& \frac{d \xi_{i}}{d t}=3 \epsilon^{3}\left[\sum_{h \neq i} \frac{\xi_{h}-\xi_{i}}{\left|\xi_{h}-\xi_{i}\right|^{3}}\left(1-\frac{R_{h}}{\bar{R}}\right)+\sum_{h \neq i} \frac{\partial \gamma}{\partial \xi_{h}}\left(1-\frac{R_{h}}{\bar{R}}\right)\right]+O\left(\epsilon^{4(1-\eta)}\right) .
\end{aligned}
$$

The error terms were derived in [4] and they are estimated here under the scaling hypotheses, size/distance $=\epsilon^{1-\eta}$. From the structure of (2.2), (2.3), we observe that in order to pass to the limit, the estimation of the terms $\epsilon \sum_{i \neq j} 1 /\left|\xi_{i}-\xi_{j}\right|, \epsilon \sum_{i} \gamma\left(\xi_{i}, \xi_{h}\right), \epsilon^{3} \sum_{h \neq i} 1 /$ $\left|\xi_{h}-\xi_{i}\right|^{2}, \epsilon^{3} \sum_{h \neq i} \partial \gamma / \partial \xi_{h}$ is essential.

2.1. The monopole approximation. In taking the limit, the number of terms in $\sum_{i \neq j} 1 /$ $\left|\xi_{i}-\xi_{j}\right|$ increases and so, for estimating it efficiently, it is necessary to take into account 


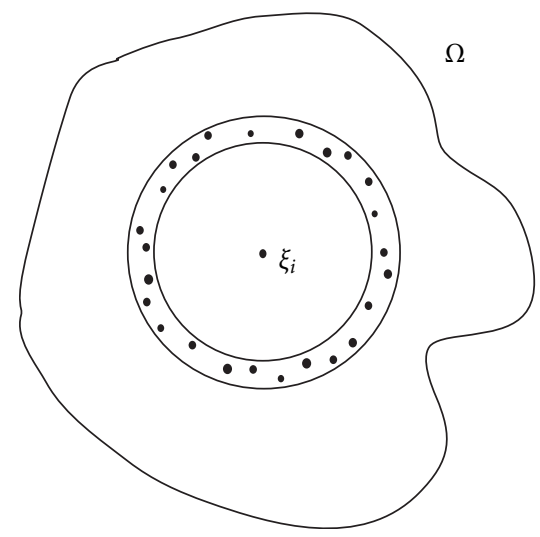

Figure 2.1

that the new particles we keep adding are further and further away. Similar considerations are needed for the other sums as well. However, the summability of this series determines the subcritical and the critical density.

We consider $\Omega \subset \mathbb{R}^{3}$ a bounded smooth domain. We take the particles initially at $t=0$ satisfying $c_{1} \epsilon^{\eta} \leq d\left(\xi_{i}, \xi_{j}\right) \leq c_{2} \epsilon^{\eta}$. Let $\xi_{i}$ be in the center of a sphere that is contained in $\Omega$ as shown in Figure 2.1, and we sum over the shell.

The number of particles in the infinitesimal shell is estimated by $\pi r^{2} \Delta r / d^{3}$ and the strength of the shell is

$$
\int_{0}^{\Lambda} \frac{\pi r^{2}}{d^{3}} \frac{1}{r} d r \leq \frac{1}{d}\left(\frac{\Lambda}{d}\right)^{2}
$$

So, we obtain the estimate

$$
\sum_{i \neq j} \frac{1}{\left|\xi_{i}-\xi_{j}\right|} \simeq \frac{1}{d}\left(\frac{\Lambda}{d}\right)^{2} .
$$

Analogously, we have the estimate

$$
\sum_{i \neq j} \frac{1}{\left|\xi_{i}-\xi_{j}\right|^{2}} \simeq \frac{\Lambda^{2}}{d^{3}}
$$

So, by utilizing (2.5), (2.6), and the scaling, we have

$$
\begin{aligned}
& \epsilon \sum_{i \neq j} \frac{1}{\left|\xi_{i}-\xi_{j}\right|} \simeq \epsilon \frac{1}{\epsilon^{\eta}}\left(\frac{1}{\epsilon^{\eta}}\right)^{2}=\frac{\epsilon}{\epsilon^{3 \eta}}=\epsilon^{1-3 \eta}, \\
& \epsilon^{3} \sum_{h \neq i} \frac{1}{\left|\xi_{h}-\xi_{i}\right|^{2}} \simeq \epsilon^{3} \frac{1}{\epsilon^{3 \eta}}=\epsilon^{3-3 \eta} .
\end{aligned}
$$


In the case where $\eta<1 / 3, \epsilon^{1-3 \eta} \rightarrow 0$, we obtain the mean field model. When $\eta=1 / 3$, $\epsilon^{1-3 \eta}$ is not negligible any more and we have the critical density. The $\xi$ equations in both the subcritical and critical cases tend to 0 as $\epsilon \rightarrow 0$.

2.2. The boundary estimate. We are also interested in estimating

$$
\sum_{i} \gamma\left(\xi_{i}, \xi_{h}\right), \quad \sum_{i \neq h} \frac{\partial \gamma}{\partial \xi_{h}}
$$

where $\gamma$ is the smooth part of the Green's function capturing the effect of the boundary

$$
\begin{gathered}
g(x, y)=\frac{1}{4 \pi|x-y|}+\gamma(x, y), \\
-\Delta_{y} g(x, y)=\delta_{x}(y)-\frac{1}{|\Omega|}, \quad x \in \Omega, y \in \Omega, \\
\frac{\partial g(x, y)}{\partial n_{y}}=0, \quad x \in \Omega, y \in \partial \Omega, \\
\int_{\Omega} g(x, y) d x=0,
\end{gathered}
$$

and $\gamma$ satisfies

$$
\begin{gathered}
-\Delta_{y} \gamma(x, y)=-\frac{1}{|\Omega|}, \quad x \in \Omega, y \in \Omega, \\
\frac{\partial \gamma(x, y)}{\partial n_{y}}=-\frac{\partial}{\partial n_{y}}\left(\frac{1}{4 \pi} \frac{1}{|x-y|}\right), \quad x \in \Omega, y \in \partial \Omega, \\
\int_{\Omega} \gamma(x, y) d y=-\int_{\Omega} \frac{1}{4 \pi} \frac{1}{|x-y|} .
\end{gathered}
$$

Claim 2.1.

$$
|\gamma(x, y)| \leq \frac{C}{\operatorname{dist}(x, \partial \Omega)}, \quad\left|\frac{\partial \gamma(x, y)}{\partial y}\right| \leq \frac{C}{\operatorname{dist}^{2}(x, \partial \Omega)}
$$

Proof. From classical elliptic theory [5] one has the desired estimates.

We take $\xi_{h}$ on the boundary and in the center of a sphere. Then

$$
\sum_{i} \gamma\left(\xi_{i}, \xi_{h}\right) \leq \frac{1}{2} \frac{1}{\operatorname{dist}\left(\xi_{i}, \partial \Omega\right)} \frac{\Lambda^{2}}{\operatorname{dist}^{2}\left(\xi_{i}, \partial \Omega\right)}, \quad \sum_{i \neq h} \frac{\partial \gamma}{\partial \xi_{h}} \leq \frac{1}{2} \frac{1}{\operatorname{dist}^{3}\left(\xi_{i}, \partial \Omega\right)}
$$

Taking into account that $\operatorname{dist}\left(\xi_{i}, \partial \Omega\right)=\epsilon^{\eta}$,

$$
\begin{aligned}
\epsilon \sum_{i} \gamma\left(\xi_{i}, \xi_{h}\right) & \simeq \frac{1}{2} \epsilon^{1-3 \eta}, \\
\epsilon^{3} \sum_{i \neq h} \frac{\partial \gamma}{\partial \xi_{h}} & \simeq \frac{1}{2} \epsilon^{3-3 \eta} .
\end{aligned}
$$


222 Continuum limits of particles interacting via diffusion

2.3. The estimate for the centers. We assume that

$$
R_{1}(0) \leq R_{2}(0) \leq \cdots \leq R_{N-1}(0) \leq R_{N}(0)
$$

and consider the system

$$
\begin{aligned}
& \frac{d R_{i}}{d t}=\frac{1}{R_{i}}\left\{\left(\frac{1}{\bar{R}}-\frac{1}{R_{i}}\right)+\epsilon\right. {\left[\frac{1}{N \bar{R}} \sum_{\substack{k, h \\
k \neq h}} \frac{R_{h}}{\left|\xi_{h}-\xi_{k}\right|}\left(\frac{R_{k}}{\bar{R}}-1\right)\right.} \\
&\left.\left.-\sum_{j \neq i} \frac{1}{\left|\xi_{j}-\xi_{i}\right|}\left(\frac{R_{j}}{\bar{R}}-1\right)\right]\right\}+O\left(\epsilon^{2(1-\eta)}\right), \\
& \frac{d \xi_{i}}{d t}=3 \epsilon^{3} \sum_{h \neq i} \frac{\xi_{h}-\xi_{i}}{\left|\xi_{h}-\xi_{i}\right|^{3}}\left(1-\frac{R_{h}}{\bar{R}}\right)+O\left(\epsilon^{4(1-\eta)}\right),
\end{aligned}
$$

for $r=O(1)$, globally in time.

Theorem 2.2. Let

$$
c \epsilon^{\eta}<\left|\xi_{i}(0)-\xi_{j}(0)\right|<\frac{4}{3} c \epsilon^{\eta}, \quad i \neq j
$$

and let $T=\epsilon^{-\lambda}, \lambda \geq 0$. Then

$$
\frac{c}{2} \epsilon^{\eta}<\left|\xi_{i}(t)-\xi_{j}(t)\right|<2 c \epsilon^{\eta}, \quad i \neq j,
$$

for $t \in\left[0, \epsilon^{-\lambda}\right]$, provided $\eta<(3-\lambda) / 6$.

Therefore, if $c \epsilon^{\eta}<\operatorname{dist}_{j}\left(\xi_{i}(0), \xi_{j}(0)\right)<(4 / 3) c \epsilon^{\eta}$, then $(c / 2) \epsilon^{\eta}<\operatorname{dist}_{j}\left(\xi_{i}(t), \xi_{j}(t)\right)<2 c \epsilon^{\eta}$. Proof. We have

$$
\begin{aligned}
\frac{d \xi_{i}}{d t}-\frac{d \xi_{j}}{d t}= & 3 \epsilon^{3}\left[\sum_{h \neq i} \frac{\xi_{h}-\xi_{i}}{\left|\xi_{h}-\xi_{i}\right|^{3}}\left(1-\frac{R_{h}}{\bar{R}}\right)-\sum_{h \neq j} \frac{\xi_{h}-\xi_{j}}{\left|\xi_{h}-\xi_{j}\right|^{3}}\left(1-\frac{R_{h}}{\bar{R}}\right)\right]+O\left(\epsilon^{4(1-\eta)}\right) \\
= & 3 \epsilon^{3}\left[\left(\sum_{h \neq i, j} \frac{\xi_{h}-\xi_{i}}{\left|\xi_{h}-\xi_{i}\right|^{3}}-\sum_{h \neq i, j} \frac{\xi_{h}-\xi_{j}}{\left|\xi_{h}-\xi_{j}\right|^{3}}\right)\left(1-\frac{R_{h}}{\bar{R}}\right)\right] \\
& +3 \epsilon^{3}\left[\frac{\xi_{j}-\xi_{i}}{\left|\xi_{j}-\xi_{i}\right|^{3}}\left(1-\frac{R_{j}}{\bar{R}}\right)-\frac{\xi_{i}-\xi_{j}}{\left|\xi_{i}-\xi_{j}\right|^{3}}\left(1-\frac{R_{i}}{\bar{R}}\right)\right]+O\left(\epsilon^{4(1-\eta)}\right) .
\end{aligned}
$$




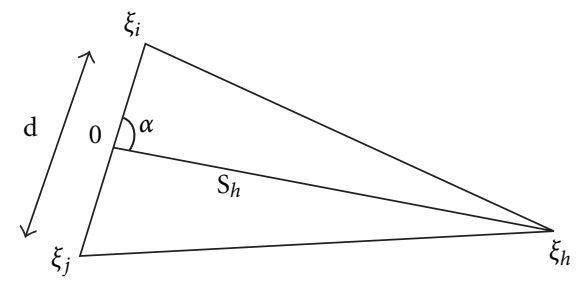

Figure 2.2. The dipole estimate.

Equivalently,

$$
\begin{aligned}
\frac{d \xi_{i}}{d t}-\frac{d \xi_{j}}{d t}= & 3 \epsilon^{3} \sum_{h \neq i, j}\left(\frac{\xi_{h}-\xi_{i}}{\left|\xi_{h}-\xi_{i}\right|^{3}}-\frac{\xi_{h}-\xi_{j}}{\left|\xi_{h}-\xi_{j}\right|^{3}}\right)\left(1-\frac{R_{h}}{\bar{R}}\right) \\
& +6 \epsilon^{3} \frac{\xi_{j}-\xi_{i}}{\left|\xi_{j}-\xi_{i}\right|^{3}}\left(1-\frac{\left(R_{i}+R_{j}\right) / 2}{\bar{R}}\right)+O\left(\epsilon^{4(1-\eta)}\right) .
\end{aligned}
$$

Moreover,

$$
\begin{aligned}
R_{i} \frac{d R_{i}}{d t} & =\left(\frac{1}{\bar{R}}-\frac{1}{R_{i}}\right)+\epsilon \varphi_{i}(R, \xi)+O\left(\epsilon^{2(1-\eta)}\right), \\
R_{j} \frac{d R_{j}}{d t} & =\left(\frac{1}{\bar{R}}-\frac{1}{R_{j}}\right)+\epsilon \varphi_{j}(R, \xi)+O\left(\epsilon^{2(1-\eta)}\right),
\end{aligned}
$$

where

$$
\varphi_{i}(R, \xi)=\frac{1}{N \bar{R}} \sum_{\substack{k, h \\ k \neq h}} \frac{R_{h}}{\left|\xi_{h}-\xi_{k}\right|}\left(\frac{R_{k}}{\bar{R}}-1\right)-\sum_{\substack{j \neq i \\ j \neq h}} \frac{1}{\left|\xi_{j}-\xi_{i}\right|}\left(\frac{R_{j}}{\bar{R}}-1\right) .
$$

So,

$$
\begin{aligned}
\frac{d}{d t}\left(\frac{R_{i}^{2}}{2}-\frac{R_{j}^{2}}{2}\right) & =\frac{1}{R_{j}}-\frac{1}{R_{i}}+\epsilon\left(\varphi_{i}-\varphi_{j}\right)+O\left(\epsilon^{2(1-\eta)}\right) \\
& =\frac{R_{i}-R_{j}}{R_{i} R_{j}}+\epsilon\left(\varphi_{i}-\varphi_{j}\right)+O\left(\epsilon^{2(1-\eta)}\right)
\end{aligned}
$$

with

$$
\varphi_{i}-\varphi_{j}=\sum_{h \neq i, j}\left(\frac{1}{\left|\xi_{h}-\xi_{j}\right|}-\frac{1}{\left|\xi_{h}-\xi_{i}\right|}\right)\left(\frac{R_{h}}{\bar{R}}-1\right)+\frac{1}{\left|\xi_{i}-\xi_{j}\right|} \frac{R_{i}-R_{j}}{\bar{R}} .
$$

We define, see Figure 2.2,

$$
\mu^{*}=\sum_{\substack{h \neq i, j \\ s_{h}>d}}\left|\frac{1}{\left|\xi_{h}-\xi_{j}\right|}-\frac{1}{\left|\xi_{h}-\xi_{i}\right|}\right|+\sum_{\substack{h \neq i, j \\ s_{h}<d}}\left|\frac{1}{\left|\xi_{h}-\xi_{j}\right|}-\frac{1}{\left|\xi_{h}-\xi_{i}\right|}\right|=\mu_{1}+\mu_{2},
$$


224 Continuum limits of particles interacting via diffusion

where

$$
s_{h}=d\left(\xi_{h}, \frac{\xi_{i}+\xi_{j}}{2}\right), \quad d=\left|\xi_{i}-\xi_{j}\right|
$$

Set

$$
\mu_{h}\left(\xi_{h}, \alpha\right)=\left|\frac{1}{\left|\xi_{h}-\xi_{j}\right|}-\frac{1}{\left|\xi_{h}-\xi_{i}\right|}\right|
$$

For $s_{h}>d$,

$$
\mu_{1 h}\left(\xi_{h}, \alpha\right) \leq M_{1} \mu_{1 h}\left(\xi_{h}, 0\right) \leq M_{1} \frac{d}{s_{h}^{2}}
$$

where

$$
\begin{aligned}
\mu_{1 h}\left(\xi_{h}, 0\right) & =\left|\frac{1}{s_{h}+d / 2}-\frac{1}{s_{h}-d / 2}\right|=\left|\frac{s_{h}-(d / 2)-s_{h}-d / 2}{s_{h}^{2}-d^{2} / 4}\right| \\
& =\left|\frac{-d}{s_{h}^{2}-d^{2} / 4}\right|=\frac{d}{s_{h}^{2}-d^{2} / 4}
\end{aligned}
$$

while for $s_{h}<d$,

$$
\mu_{2 h}\left(\xi_{h}, \alpha\right) \leq M_{2} \frac{1}{s_{h}}
$$

where $M_{1}, M_{2}>0$ are suitable constants. Then, from (2.25),

$$
\left|\varphi_{i}-\varphi_{j}\right| \leq\left(\mu_{1}+\mu_{2}\right)\left|\frac{R_{h}}{\bar{R}}-1\right|+\frac{1}{\left|\xi_{i}-\xi_{j}\right|}\left|\frac{R_{i}-R_{j}}{\bar{R}}\right| .
$$

Equation (2.21) can be written as

$$
\begin{aligned}
\frac{d}{d t}\left(\left|\xi_{i}-\xi_{j}\right|\right)= & 3 \epsilon^{3} \sum_{h \neq i, j}\left(\frac{\left\langle\xi_{h}-\xi_{i}, \xi_{i}-\xi_{j}\right\rangle}{\left|\xi_{h}-\xi_{i}\right|^{3}\left|\xi_{i}-\xi_{j}\right|}-\frac{\left\langle\xi_{h}-\xi_{j}, \xi_{i}-\xi_{j}\right\rangle}{\left|\xi_{h}-\xi_{j}\right|^{3}\left|\xi_{i}-\xi_{j}\right|}\right)\left(1-\frac{R_{h}}{\bar{R}}\right) \\
& -6 \epsilon^{3} \frac{1}{\left|\xi_{i}-\xi_{j}\right|^{2}}\left(1-\frac{\left(R_{i}+R_{j}\right) / 2}{\bar{R}}\right)+O\left(\epsilon^{4(1-\eta)}\right) .
\end{aligned}
$$


Set

$$
\begin{aligned}
\sigma_{h}\left(\xi_{h}, \alpha\right) & =\left|\frac{\left\langle\xi_{h}-\xi_{i}, \xi_{i}-\xi_{j}\right\rangle}{\left|\xi_{h}-\xi_{i}\right|^{3}\left|\xi_{i}-\xi_{j}\right|}-\frac{\left\langle\xi_{h}-\xi_{j}, \xi_{i}-\xi_{j}\right\rangle}{\left|\xi_{h}-\xi_{j}\right|^{3}\left|\xi_{i}-\xi_{j}\right|}\right| \\
\sigma^{*} & =3 \epsilon^{3}\left(\sum_{\substack{h \neq i, j \\
s_{h}>d}}+\sum_{\substack{h \neq i, j \\
s_{h}<d}}\right)\left|\frac{\left\langle\xi_{h}-\xi_{i}, \xi_{i}-\xi_{j}\right\rangle}{\left|\xi_{h}-\xi_{i}\right|^{3}\left|\xi_{i}-\xi_{j}\right|}-\frac{\left\langle\xi_{h}-\xi_{j}, \xi_{i}-\xi_{j}\right\rangle}{\left|\xi_{h}-\xi_{j}\right|^{3}\left|\xi_{i}-\xi_{j}\right|}\right| \\
& =3 \epsilon^{3}\left(\sum_{\substack{h \neq i, j \\
s_{h}>d}}+\sum_{\substack{h \neq i, j \\
s_{h}<d}}\right) \sigma_{h}\left(\xi_{h}, \alpha\right) .
\end{aligned}
$$

Then, for $s_{h}>d$,

$$
\sigma_{1 h}\left(\xi_{h}, \alpha\right) \leq K_{1} \sigma_{1 h}\left(\xi_{h}, 0\right) \leq K_{1} \frac{d}{s_{h}^{3}}
$$

where

$$
\begin{aligned}
\sigma_{1 h}\left(\xi_{h}, 0\right) & =\left|\frac{\left(s_{h}-d / 2\right) d}{\left(s_{h}-d / 2\right)^{3} d}-\frac{\left(s_{h}+d / 2\right) d}{\left(s_{h}+d / 2\right)^{3} d}\right| \\
& =\left|\frac{1}{\left(s_{h}-d / 2\right)^{2}}-\frac{1}{\left(s_{h}+d / 2\right)^{2}}\right|=\frac{2 d s_{h}}{\left(s_{h}^{2}-d^{2} / 4\right)^{2}},
\end{aligned}
$$

and for $s_{h}<d$,

$$
\sigma_{2 h}\left(\xi_{h}, \alpha\right) \leq K_{2} \frac{1}{s_{h}^{2}}
$$

By utilizing the estimate (from Lemma 3.2(iii))

$$
\left|\frac{R_{h}}{\bar{R}}\right|<C \sup R_{h}{ }^{3}
$$

via (2.35), (2.36), and (2.38), we obtain the following estimate:

$$
\begin{aligned}
I & :=3 \epsilon^{3} \sum_{\substack{h \neq i, j \\
s_{h}>d}} \sigma_{1 h}\left(\xi_{h}, \alpha\right)\left|\left(1-\frac{R_{h}}{\bar{R}}\right)\right|+3 \epsilon^{3} \sum_{\substack{h \neq i, j \\
s_{h}<d}} \sigma_{2 h}\left(\xi_{h}, \alpha\right)\left|\left(1-\frac{R_{h}}{\bar{R}}\right)\right| \\
& \leq 3 \epsilon^{3} K \sup R_{h}^{3}\left[d \sum_{\substack{h \neq i, j \\
s_{h}>d}} \frac{1}{s_{h}^{3}}+\sum_{\substack{h \neq i, j \\
s_{h}<d}} \frac{1}{s_{h}^{2}}\right] .
\end{aligned}
$$

By continuous dependence, (2.18), and the structure of the $\xi$-equations, there is $T_{0}>0$ depending only on $c$ and $\eta$, such that

$$
\frac{c}{2} \epsilon^{\eta}<\left|\xi_{k}(t)-\xi_{l}(t)\right|<2 c \epsilon^{\eta}, \quad k \neq l,
$$


for $t \in\left[0, T_{0}\right]$. Let $T_{0}$ be the maximal time for which (2.41) holds. The estimates below are for $t \in\left[0, T_{0}\right]$.

We are going to estimate

$$
\sum_{\substack{h \neq i, j \\ s_{h}>d}} \frac{1}{d^{3}\left(\xi_{h},\left(\xi_{i}+\xi_{j}\right) / 2\right)}, \quad \sum_{\substack{h \neq i, j \\ s_{h}<d}} \frac{1}{d^{2}\left(\xi_{h},\left(\xi_{i}+\xi_{j}\right) / 2\right)} .
$$

We have

$$
\sum_{\substack{h \neq i, j \\ s_{h}>d}} \frac{1}{d^{3}\left(\xi_{h},\left(\xi_{i}+\xi_{j}\right) / 2\right)} \leq\left(\frac{2}{c}\right)^{3} \frac{1}{\epsilon^{3 \eta}} \sum_{\substack{h \neq i, j \\ s_{h}>d}} \frac{1}{d^{3}(h,(i+j) / 2)}
$$

This step is a normalization, scaling out the distance and summing over a lattice. In what follows, we are imagining the point $\left(\xi_{i}+\xi_{j}\right) / 2$ in the center of a sphere that encloses $\Omega$. There are universal constants involved here that we ignore since they will not affect the estimation. We compute

$$
\sum_{\substack{h \neq i, j \\ s_{h}>d}} \frac{1}{d^{3}(h,(i+j) / 2)} \leq \int_{1}^{2 \Lambda / c \epsilon^{\eta}} \frac{1}{r^{3}} r^{2} d r \leq \ln \left(\frac{2 \Lambda}{c \epsilon^{\eta}}\right) .
$$

Analogously,

$$
\begin{gathered}
\sum_{\substack{h \neq i, j \\
s_{h}<d}} \frac{1}{d^{2}\left(\xi_{h},\left(\xi_{i}+\xi_{j}\right) / 2\right)} \leq\left(\frac{2}{c}\right)^{3} \frac{1}{\epsilon^{3 \eta}} \sum_{\substack{h \neq i, j \\
s_{h}<d}} \frac{1}{d^{2}(h,(i+j) / 2)}, \\
\sum_{\substack{h \neq i, j \\
s_{h}<d}} \frac{1}{d^{2}(h,(i+j) / 2)} \leq \int^{2 c \epsilon^{\eta}} \frac{1}{r^{2}} r^{2} d r \leq 2 c \epsilon^{\eta} .
\end{gathered}
$$

Taking into account that

$$
R_{h}{ }^{3} \leq\left(\frac{2 \Lambda}{c \epsilon^{\eta}}\right)^{3}, \quad d\left(\xi_{i}, \xi_{j}\right) \leq 2 c \epsilon^{\eta}
$$

we obtain from (2.40), via (2.43), (2.44), (2.45), and (2.46), the following estimate:

$$
I \leq 3 K 2^{7} \Lambda^{3} c^{-5} \epsilon^{3-5 \eta}+3 K 2^{7} \Lambda^{3} c^{-5} \epsilon^{3-5 \eta} \ln \left(\frac{2 \Lambda}{c \epsilon^{\eta}}\right) .
$$

Next we consider the second term in (2.33). By (2.39) and (2.46),

$$
6 \epsilon^{3} \frac{1}{\left|\xi_{i}-\xi_{j}\right|^{2}}\left(1-\frac{\left(R_{i}+R_{j}\right) / 2}{\bar{R}}\right) \leq 6 \epsilon^{3} \frac{1}{\left|\xi_{i}-\xi_{j}\right|^{2}}\left(\frac{2 \Lambda}{c \epsilon^{\eta}}\right)^{3} .
$$


Thus, from (2.33), we obtain via (2.46), (2.47),

$$
\begin{aligned}
\frac{d}{d t} d \geq & -3 K \cdot 2^{7} \Lambda^{3} c^{-5} \epsilon^{3-5 \eta}-3 K \cdot 2^{7} \Lambda^{3} c^{-5} \epsilon^{3-5 \eta} \ln \left(\frac{2 \Lambda}{c \epsilon^{\eta}}\right) \\
& -6 \cdot 2^{3} \Lambda^{3} c^{-3} \epsilon^{3-3 \eta} \frac{1}{d^{2}}+O\left(\epsilon^{4(1-\eta)}\right) .
\end{aligned}
$$

By ignoring the second and the error terms in (2.49), we have

$$
\begin{gathered}
\frac{d}{d t} d \geq-(\text { Const. }) \epsilon^{3-3 \eta} \frac{1}{d^{2}}, \quad t \in\left[0, T_{0}\right], \\
d^{2} \cdot \frac{d}{d t} d \geq-(\text { Const. }) \epsilon^{3-3 \eta}, \\
\left.d^{3}(t) \geq d^{3}(0)-\text { (Const. }\right) \epsilon^{3-3 \eta} t .
\end{gathered}
$$

Hence we need

$$
\text { (Const.) } \epsilon^{3-3 \eta} T_{0}<\epsilon^{3 \eta}
$$

so that (2.41) is never violated.

Let $T_{0}=\epsilon^{-\lambda}$. We need

$$
3-3 \eta-\lambda>3 \eta \Longleftrightarrow \eta<\frac{3-\lambda}{6} .
$$

With analogous arguments, also an upper bound is established. Hence the estimate (2.41) holds for $t \in\left[0, \epsilon^{-\lambda}\right]$. In order to estimate $\left|\varphi_{i}-\varphi_{j}\right|$, we return back and examine (2.32). As soon as (2.29), (2.31) hold with similar estimates as (2.43), (2.44), and (2.45), we derive

$$
\begin{aligned}
& \mu_{1} \leq d \sum_{\substack{h \neq i, j \\
s_{h}>d}} \frac{1}{d^{2}\left(\xi_{h},\left(\xi_{i}+\xi_{j}\right) / 2\right)} \leq d\left(\frac{2}{c}\right)^{3} \frac{1}{\epsilon^{3 \eta}} \frac{2 \Lambda}{c \epsilon^{\eta}}=2^{4} c^{-4} \epsilon^{-3 \eta} \Lambda, \\
& \mu_{2} \leq \sum_{\substack{h \neq i, j \\
s_{h}<d}} \frac{1}{d\left(\xi_{h},\left(\xi_{i}+\xi_{j}\right) / 2\right)} \leq\left(\frac{2}{c}\right)^{3} \frac{1}{\epsilon^{3 \eta}} 2^{2} c^{2} \epsilon^{2 \eta} \leq 2^{5} c^{-1} \epsilon^{\eta} .
\end{aligned}
$$

So, utilizing (2.32), (2.39), and (2.46),

$$
\left|\varphi_{i}-\varphi_{j}\right| \leq c \epsilon^{-3 \eta}\left(\frac{2 \Lambda}{c \epsilon^{\eta}}\right)^{3} \leq M \epsilon^{-6 \eta}
$$

\section{Passage to the limit}

Set

$$
d \mu_{t}^{\epsilon}(R, \xi)=\epsilon^{3 \eta} \sum_{i \in N} \delta_{R_{i}^{\epsilon}(t), \xi_{i}^{\epsilon}(t)}(R, \xi)
$$


228 Continuum limits of particles interacting via diffusion

where $d \mu_{t}^{\epsilon}$ is an $O(1)$ quantity, and is defined via

$$
\left\langle z, d \mu_{t}^{\epsilon}\right\rangle=\int z d \mu_{t}^{\epsilon}=\epsilon^{3 \eta} \sum_{i} z\left(R_{i}^{\epsilon}(t), \xi_{i}^{\epsilon}(t)\right) .
$$

The Riesz representation theorem provides the existence of a unique positive Borel, and regular measure $\mu_{t} \epsilon \in\left(C_{c}\right)^{*}$ where $z \in C_{c}(\Omega \times(0, \infty))$ with compact support in $\Omega \times$ $(0, \infty)$. We begin with some lemmas from Niethammer and Otto $[7,8]$.

Lemma 3.1. The following hold true:

(i) $\int d \mu_{t}^{\epsilon} \leq 1, t \in[0, \infty)$;

(ii) $\int R^{3} d \mu_{t}^{\epsilon}=1, t \in[0, \infty)$;

(iii) for $M>0, T>0, R^{*}>0$, $\sup _{t \in(0, T)} \int_{R>R^{*}} R^{3} d \mu_{t}^{\epsilon}<M$, uniformly in $\epsilon$.

Proof. (i) From the definition of the measure, we have

$$
\int z d \mu_{t}^{\epsilon}=\epsilon^{3 \eta} \sum_{i} z\left(R_{i}^{\epsilon}(t), \xi_{i}^{\epsilon}(t)\right)
$$

So, for $z=1$,

$$
\begin{aligned}
\int d \mu_{t}^{\epsilon} & =\epsilon^{3 \eta} \sum_{i} 1=\epsilon^{3 \eta} \cdot(\text { number of particles at time } t) \\
& \leq \epsilon^{3 \eta} \cdot(\text { number of particles initially })=1
\end{aligned}
$$

(ii) Again, from the definition of the measure for $z=R^{3}$, we have

$$
\int z d \mu_{t}^{\epsilon}=\int R^{3} d \mu_{t}^{\epsilon}=\epsilon^{3 \eta} \sum_{i} R_{i}^{3}(t)=\epsilon^{3 \eta} \sum_{i} R_{i}{ }^{3}(0)=1 .
$$

(iii) Let $\vartheta=\vartheta(R)$ be a smooth cut-off function with $\vartheta(R)=1$ for $R>R^{*}, \vartheta(R)=0$ for $R<R^{*} / 2$ and $0<\vartheta^{\prime}(R) \leq 3 / R^{*}$. We calculate

$$
\left|\frac{d}{d t} \int \vartheta R^{3} d \mu_{t}^{\epsilon}\right|=\left|\epsilon^{3 \eta} \sum_{i}\left(\vartheta^{\prime}\left(R_{i}\right) R_{i}{ }^{3}+3 \eta R_{i}{ }^{2}\right) \dot{R}_{i}\right| \leq \frac{C}{R^{*}}\left(\epsilon^{3 \eta} \sum_{i} R_{i}^{3} \dot{R}_{i}^{2}\right)^{1 / 2},
$$

and hence

$$
\int_{R>R^{*}} R^{3} d \mu_{t}^{\epsilon} \leq \int \vartheta R^{3} d \mu_{t}^{\epsilon} \leq M
$$

For the sake of simplicity in what follows, we use the same notation for a sequence and for its subsequence.

Lemma 3.2. The following hold true:

(i) $d \mu_{t}^{\epsilon} \rightarrow d \mu_{t}$, in $\left(C_{c}\right)^{*}$;

(ii) there exist $1 / \bar{R} \in L^{\infty}(0, T) \bigcap B V(0, T)$ such that $1 / \bar{R}^{\epsilon} \rightarrow 1 / \bar{R}$, in $L^{p}(0, T), 1 \leq p<\infty$; 
(iii) $\left|R_{j}{ }^{\epsilon}(t) / \bar{R}^{\epsilon}(t)\right| \leq C \sup \left(R_{j}{ }^{\epsilon}(t)\right)^{3}$, uniformly in $\epsilon$, with $C$ independent of $\epsilon$, and depending only on the initial distribution;

(iv) $\bar{R}=\int R d \mu_{t} / \int d \mu_{t}$;

(v) $\int z d \mu_{t}^{\epsilon} \rightarrow \int z d \mu_{t}$, uniformly in $t \in[0, \infty)$.

Proof. (i) From (3.2),

$$
\left\langle z, d \mu_{t}^{\epsilon}\right\rangle=\int z d \mu_{t}^{\epsilon}=\epsilon^{3 \eta} \sum_{i} z\left(R_{i}^{\epsilon}(t), \xi_{i}^{\epsilon}(t)\right)
$$

As soon as

$$
\left|\left\langle z, d \mu_{t}^{\epsilon}\right\rangle\right| \leq \max _{R, \xi} \epsilon^{3 \eta}\left|\sum_{i} z\left(R_{i}^{\epsilon}(t), \xi_{i}^{\epsilon}(t)\right)\right| \leq C
$$

we can choose a subsequence such that

$$
d \mu_{t}^{\epsilon} \stackrel{*}{\longrightarrow} d \mu_{t}, \quad \text { in }\left(C_{c}\right)^{*}
$$

(ii) We know the compact embedding $B V$ into $L^{1}, B V$ into $L^{p}$. If we show that $1 / \bar{R}^{\epsilon}$ is uniformly bounded in $1 / \bar{R} \in L^{\infty}(0, T) \bigcap B V(0, T)$, then we are going to have strong convergence of a subsequence of $1 / \bar{R}^{\epsilon}$ in $L^{p}$. Note that

$$
\frac{1}{\bar{R}^{\epsilon}}=\frac{N \epsilon^{3 \eta}}{\sum_{i} \epsilon^{3 \eta} R_{i}^{\epsilon}(t)}
$$

We are going to show that

$$
\frac{1}{\bar{R}^{\epsilon}} \leq C \sup _{k} R_{k}^{\epsilon}(t)
$$

By the interpolation fact,

$$
\left\|R^{\epsilon}\right\|_{l_{3}} \leq\left\|R^{\epsilon}\right\|_{l_{\infty}}^{2 / 3}\left\|R^{\epsilon}\right\|_{l_{1}}^{1 / 3}, \quad \sum_{k} R_{k}^{\epsilon 3} \leq\left(\sup R_{k}^{\epsilon 2}\right) \sum_{k} R_{k}^{\epsilon}, \quad \frac{1}{\sum_{k} R_{k}^{\epsilon}} \leq \frac{\sup R_{k}^{\epsilon 2}}{\sum_{k} R_{k}^{\epsilon 3}} .
$$

We have

$$
\begin{aligned}
\frac{1}{\bar{R}^{\epsilon}} & =\frac{N \epsilon^{3 \eta}}{\sum_{k} \epsilon^{3 \eta} R_{k}^{\epsilon}(t)} \leq \frac{N \epsilon^{3 \eta} \sup _{k} R_{k}^{\epsilon 2}}{\sum_{k} \epsilon^{3 \eta} R_{k}^{\epsilon 3}(t)} \\
& \leq \frac{C \sup _{k} R_{k}^{\epsilon 2}(t)}{\sum_{k} \epsilon^{3 \eta} R_{k}^{\epsilon 3}(t)}=\frac{C \sup _{k} R_{k}^{\epsilon 2}(t)}{\sum_{k} \epsilon^{3 \eta} R_{k}^{\epsilon 3}(0)}, \quad N \epsilon^{3 \eta}=O(1) .
\end{aligned}
$$


From the definition of measure describing initial data, we conclude that

$$
\frac{1}{\bar{R}^{\epsilon}} \leq C \sup _{k} R_{k}^{\epsilon 2}(t)
$$

So,

$$
\frac{1}{\bar{R}^{\epsilon}} \quad \text { in } L^{\infty}(0, T)
$$

It is known that $N \epsilon^{3 \eta}$ is a function of bounded variation. We would like to show that $\sum_{i} \epsilon^{3 \eta} R_{i}^{\epsilon}(t)$ is also a function of bounded variation. Indeed,

$$
\left\|\frac{d}{d t} \sum_{i} \epsilon^{3 \eta} R_{i}^{\epsilon}\right\|_{L^{1}(0, T)}=\left\|\sum_{i} \epsilon^{3 \eta} \dot{R}_{i}^{\epsilon}\right\|_{L^{1}(0, T)} \leq \sup _{i}\left\|\dot{R}_{i}^{\epsilon}\right\|_{L^{1}(0, T)} \leq C .
$$

(It is known (see [3]) that at the extinction time $R(t)=(T-t)^{1 / 3}$, while $\dot{R}_{i}=1 / R_{i}{ }^{2}=$ $1 /(T-t)^{2 / 3}$.) So, the desired quotient is a function of bounded variation.

(iii) Follows immediately from (ii).

(iv) From the definition of the measure, we have

$$
\int \frac{R}{\bar{R}^{\epsilon}} d \mu_{t}^{\epsilon}=\epsilon^{3 \eta} \frac{1}{\bar{R}^{\epsilon}} \sum_{i} R_{i}^{\epsilon}(t)=\epsilon^{3 \eta} \sum_{i} 1=\int d \mu_{t}^{\epsilon}
$$

By utilizing (i), (ii), we have the desired expression for $\bar{R}$.

(v) We fix $t_{1}, t_{2}$ and we calculate

$$
\begin{aligned}
\left|\int z d \mu_{t_{1}}^{\epsilon}-\int z d \mu_{t_{2}}^{\epsilon}\right| \leq & \left\{\left|t_{1}-t_{2}\right|\left(\int_{0}^{\infty}\left|\frac{d}{d t} \int z d \mu_{t}^{\epsilon}\right|^{2} d t\right)\right\}^{1 / 2} \\
\leq & \left|t_{1}-t_{2}\right|^{1 / 2}\left(\int_{0}^{\infty}\left|\frac{d}{d t}\left(\epsilon^{3 \eta} \sum_{i} z\left(R_{i}^{\epsilon}, \xi_{i}^{\epsilon}\right)\right)\right|^{2}\right) \\
\leq & \left|t_{1}-t_{2}\right|^{1 / 2} \int_{0}^{\infty} \epsilon^{3 \eta} \sum_{i} \frac{\partial z}{\partial R}\left(R_{i}^{\epsilon}(t), \xi_{i}^{\epsilon}(t)\right) \dot{R}_{i}^{\epsilon}(t) \\
& +\epsilon^{3 \eta} \sum_{i} \frac{\partial z}{\partial \xi}\left(R_{i}^{\epsilon}(t), \xi_{i}^{\epsilon}(t)\right) \dot{\xi}_{i}^{\epsilon}(t) \\
\leq & \left|t_{1}-t_{2}\right|^{1 / 2} \sup \left|\frac{\partial z}{\partial R}\right| \frac{1}{R^{3}} .
\end{aligned}
$$

So, from Lemma 3.1(i), the assertion follows.

Our aim is the passage to the limit as $\epsilon \rightarrow 0$ and the derivation of the conservation law in a weak sense. 
Theorem 3.3. Let $z \in C_{c}(\Omega \times(0, \infty))$, and $\mu_{t} \in\left(C_{c}\right)^{*}$.

Then, as $\epsilon \rightarrow 0$, the conservation law is satisfied:

$$
\int_{0}^{T} \int\left(\frac{\partial z}{\partial t}+J \frac{\partial z}{\partial R}\right) d \mu_{t} d t=0, \quad \text { uniformly in } t \in[0, \infty)
$$

where

$$
J=\frac{d R}{d t}=\left\{\begin{array}{l}
\frac{1}{R}\left(\frac{1}{\bar{R}}-\frac{1}{R}\right), \quad \eta<\frac{1}{3}, \\
\frac{1}{R}\left\{\left(\frac{1}{\bar{R}}-\frac{1}{R}\right)+K(t)-\int\left(\frac{1}{|\xi-\eta|}+4 \pi \gamma(\xi, \eta)\right)\left(\frac{R}{\bar{R}}-1\right) d \mu_{t}\right\}, \quad \eta=\frac{1}{3},
\end{array}\right.
$$

where $K(t)$ is such that $(d / d t) \int R^{3} d \mu_{t}=0$.

Proof. The following hold:

$$
\begin{gathered}
\int_{0}^{T} \frac{d}{d t} \epsilon^{3 \eta} \sum_{i} z\left(R_{i}{ }^{\epsilon}(t), \xi_{i}{ }^{\epsilon}(t)\right) d t=0, \\
\int_{0}^{T} \sum_{i} \epsilon^{3 \eta} \frac{d}{d t} z\left(R_{i}{ }^{\epsilon}(t), \xi_{i}^{\epsilon}(t)\right) d t=0, \\
\int_{0}^{T} \sum_{i} \epsilon^{3 \eta}\left(\frac{\partial z}{\partial t}\left(R_{i}{ }^{\epsilon}(t), \xi_{i}^{\epsilon}(t)\right)+\frac{\partial z}{\partial R}\left(R_{i}^{\epsilon}(t), \xi_{i}{ }^{\epsilon}(t)\right) \dot{R}_{i}{ }^{\epsilon}(t)+\frac{\partial z}{\partial \xi}\left(R_{i}{ }^{\epsilon}(t), \xi_{i}{ }^{\epsilon}(t)\right) \dot{\xi}_{i}^{\epsilon}(t)\right) d t=0 .
\end{gathered}
$$

We distinguish between two cases: (I) the subcritical for $\eta<1 / 3$ and (II) the critical for $\eta=1 / 3$.

(I) Subcritical case

$$
\begin{gathered}
\int_{0}^{T} \sum_{i} \epsilon^{3 \eta} \frac{\partial z}{\partial t}\left(R_{i}{ }^{\epsilon}(t), \xi_{i}^{\epsilon}(t)\right) d t+\int_{0}^{T} \sum_{i} \epsilon^{3 \eta} \frac{\partial z}{\partial R}\left(R_{i}{ }^{\epsilon}(t), \xi_{i}{ }^{\epsilon}(t)\right) \dot{R}_{i}{ }^{\epsilon}(t) d t \\
+\int_{0}^{T} \sum_{i} \epsilon^{3 \eta} \frac{\partial z}{\partial \xi}\left(R_{i}^{\epsilon}(t), \xi_{i}{ }^{\epsilon}(t)\right) \dot{\xi}_{i}^{\epsilon}(t) d t=0 .
\end{gathered}
$$

From (3.2),

$$
\int_{0}^{T} \int \frac{\partial z}{\partial t} d \mu_{t}{ }^{\epsilon} d t+\int_{0}^{T}\left(\int \frac{\partial z}{\partial R} d \mu_{t}{ }^{\epsilon}\right) \dot{R}_{i}{ }^{\epsilon}(t) d t+\int_{0}^{T}\left(\int \frac{\partial z}{\partial \xi} d \mu_{t}{ }^{\epsilon}\right) \dot{\xi}_{i}{ }^{\epsilon}(t) d t=0
$$

By utilizing the expression of the R-equations (2.2) and the estimates (2.7), (2.14), we observe that the terms $\epsilon \sum_{i \neq j}\left(1 /\left|\xi_{i}-\xi_{j}\right|\right), \epsilon \sum_{i} \gamma\left(\xi_{i}, \xi_{h}\right)$ tend to zero as $\epsilon \rightarrow 0$. Moreover, for the $\xi$ equations $(2.3)$, by (2.8), (2.15), the corresponding terms tend to zero as well. 
232 Continuum limits of particles interacting via diffusion

The error term, for example, for the $R$-equations is of the form

$$
\begin{aligned}
& O\left(\sum_{i \neq h} \frac{\epsilon R_{h} \epsilon\left\|r_{h}\right\|_{C^{3+\alpha}\left(S^{2}\right)}}{\left|\xi_{i}-\xi_{h}\right|}+\frac{\epsilon^{2}\left(R_{i}+R_{h}\right)\left\|r_{h}\right\|_{C^{3+\alpha}\left(S^{2}\right)}}{\left|\xi_{i}-\xi_{h}\right|^{2}}\right. \\
& \quad+\sum_{i \neq h} \frac{\epsilon^{2}\left(R_{i}^{2}+R_{h}^{2}\right)}{\left|\xi_{i}-\xi_{h}\right|^{3}} \sum_{i \neq h} \frac{\epsilon^{2}\left(R_{h}\left\|r_{i}\right\|_{C^{3+\alpha}\left(S^{2}\right)}+R_{h}\left\|r_{h}\right\|_{C^{3+\alpha}\left(S^{2}\right)}\right)}{\left|\xi_{i}-\xi_{h}\right|^{2}} \\
& \left.\quad+\sum_{i} \gamma\left(\xi_{i}, \xi_{h}\right) \epsilon R_{i} \epsilon\left\|r_{i}\right\|_{C^{3+\alpha}\left(S^{2}\right)}+\sum_{i} \epsilon^{2} R_{i}{ }^{2} \frac{\partial^{2} \gamma}{\partial \xi_{i}^{2}}+\sum_{i} \epsilon^{2} R_{i}\left\|r_{i}\right\|_{C^{3+\alpha}\left(S^{2}\right)} \frac{\partial \gamma}{\partial \xi_{i}}+\epsilon^{2}\left\|r_{i}\right\|_{C^{3+\alpha}\left(S^{2}\right)}^{2}\right)
\end{aligned}
$$

which also tends to zero utilizing the basic estimate. So, by Lemma 3.2(ii), (iii), in the limit as $\epsilon \rightarrow 0$, we obtain

$$
\frac{d}{d t} R_{i}^{\epsilon}(t) \longrightarrow J=\frac{1}{R}\left(\frac{1}{R}-\frac{1}{R}\right)
$$

Hence, the expected conservation law is an immediate consequence of (3.24) and Lemma $3.2(\mathrm{v})$.

(II) Critical case. It is important to note that for $\eta=1 / 3$,

$$
d \mu_{t}^{\epsilon}(R, \xi)=\epsilon \sum_{i \in N} \delta_{R_{i}^{\epsilon}(t), \xi_{i}^{\epsilon}(t)}(R, \xi)
$$

This scaling fact explains why $\eta=1 / 3$ is special in evaluating terms like $\epsilon \sum_{i \neq j}\left(1 / \mid \xi_{i}-\right.$ $\left.\xi_{j} \mid\right)\left(R_{j} / \bar{R}-1\right)$.

We have

$$
\begin{aligned}
\int_{0}^{T} \epsilon & \sum_{i} \frac{\partial z}{\partial t}\left(R_{i}{ }^{\epsilon}(t), \xi_{i}{ }^{\epsilon}(t)\right) d t+\int_{0}^{T} \epsilon \sum_{i} \frac{\partial z}{\partial R}\left(R_{i}{ }^{\epsilon}(t), \xi_{i}{ }^{\epsilon}(t)\right) \dot{R}_{i}{ }^{\epsilon}(t) d t \\
& +\int_{0}^{T} \epsilon \sum_{i} \frac{\partial z}{\partial \xi}\left(R_{i}{ }^{\epsilon}(t), \xi_{i}{ }^{\epsilon}(t)\right) \dot{\xi}_{i}^{\epsilon}(t) d t=0 .
\end{aligned}
$$

We are going to pass to the limit in (2.2), that is,

$$
\frac{d}{d t} R_{i}^{\epsilon}(t) \longrightarrow \frac{1}{R}\left\{\left(\frac{1}{\bar{R}}-\frac{1}{R}\right)+K(t)-\int\left(\frac{1}{|\xi-\eta|}+4 \pi \gamma(\xi, \eta)\right)\left(\frac{R}{\bar{R}}-1\right) d \mu_{t}\right\}
$$

In view of the arguments in (I) above, we only need to prove that

$$
\int \theta\left(\frac{1}{|\xi-\eta|}+4 \pi \gamma(\xi, \eta)\right)\left(\frac{R}{\bar{R}^{\epsilon}}-1\right) d \mu_{t}^{\epsilon} \longrightarrow \int \theta\left(\frac{1}{|\xi-\eta|}+4 \pi \gamma(\xi, \eta)\right)\left(\frac{R}{\bar{R}}-1\right) d \mu_{t}
$$




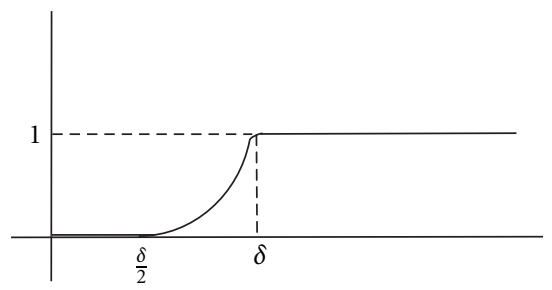

Figure 3.1. Graph of the function $\theta(\eta)$.

as $\epsilon \rightarrow 0$, uniformly in $\delta$, where

$$
\begin{aligned}
& \int \theta\left(\frac{1}{|\xi-\eta|}+4 \pi \gamma(\xi, \eta)\right)\left(\frac{R}{\bar{R}}-1\right) d \mu_{t} \\
& \quad=\lim _{\epsilon \rightarrow 0} \epsilon\left[\sum_{i \neq j} \frac{1}{\left|\xi_{i}-\xi_{j}\right|}\left(\frac{R_{j}}{\bar{R}}-1\right)+\sum_{h} 4 \pi \gamma\left(\xi_{i}, \xi_{h}\right)\left(\frac{R_{h}}{\bar{R}}-1\right)\right] .
\end{aligned}
$$

We define the test function

$$
\zeta_{i}(\eta, R)=\left(\frac{1}{\left|\xi_{i}-\eta\right|}+4 \pi \gamma\left(\xi_{i}, \eta\right)\right)\left(\frac{R}{\bar{R}}-1\right) \theta(\eta)
$$

where $\theta$ is defined as in Figure 3.1.

So,

$$
\begin{aligned}
\int \zeta_{i} d \mu_{t}{ }^{\epsilon}= & \epsilon\left[\sum_{i \neq j} \frac{1}{\left|\xi_{i}-\xi_{j}\right|}\left(\frac{R_{j}}{\bar{R}}-1\right)+4 \pi \sum_{h} \gamma\left(\xi_{i}, \xi_{h}\right)\left(\frac{R_{h}}{\bar{R}}-1\right)\right] \\
& +\sum_{d\left(\xi_{j}, \partial \Omega\right) \leq \delta}\left(\frac{1}{\left|\xi_{i}-\xi_{j}\right|}+4 \pi \gamma\left(\xi_{i}, \xi_{h}\right)\right)\left(\frac{R_{j}}{\bar{R}}-1\right) \theta \\
& +\sum_{d\left(\xi_{i}, \xi_{j}\right) \leq \delta}\left(\frac{1}{\left|\xi_{i}-\xi_{j}\right|}+4 \pi \gamma\left(\xi_{i}, \xi_{h}\right)\right)\left(\frac{R_{j}}{\bar{R}}-1\right) \theta .
\end{aligned}
$$

Utilizing the basic estimate, the last two terms of (3.33) in terms of $\delta$ are of the order of magnitude of $o(1)$. So,

$$
\int \zeta_{i} d \mu_{t}{ }^{\epsilon}=\epsilon\left[\sum_{i \neq j} \frac{1}{\left|\xi_{i}-\xi_{j}\right|}\left(\frac{R_{j}}{\bar{R}}-1\right)+4 \pi \sum_{h} \gamma\left(\xi_{i}, \xi_{h}\right)\left(\frac{R_{h}}{\bar{R}}-1\right)\right]+o(1) .
$$

From Lemma 3.2(v) as $\epsilon \rightarrow 0$,

$$
\begin{aligned}
\int \zeta_{i} d \mu_{t}{ }^{\epsilon} \longrightarrow \int \zeta_{i} d \mu_{t}+o(1)= & \int_{\substack{d(\eta, \partial \Omega)>\delta \\
d(\eta, \xi)>\delta}}\left(\frac{1}{|\xi-\eta|}+4 \pi \gamma(\xi, \eta)\right)\left(\frac{R}{\bar{R}}-1\right) d \mu_{t} \\
& +\int_{\mathcal{N}(\partial \Omega, \delta)}\left(\frac{1}{|\xi-\eta|}+4 \pi \gamma(\xi, \eta)\right)\left(\frac{R}{\bar{R}}-1\right) d \mu_{t}+o(1),
\end{aligned}
$$


234 Continuum limits of particles interacting via diffusion

where $\mathcal{N}(\partial \Omega, \delta)=\{\eta: \operatorname{dist}(\eta, \partial \Omega) \leq \delta\}$. In order to obtain (3.31), we need to show that

$$
\int_{\mathcal{N}(\partial \Omega, \delta)}\left(\frac{1}{|\xi-\eta|}+4 \pi \gamma(\xi, \eta)\right) d \mu_{t} \leq o(1)
$$

Consider $\xi \in \Omega$ and

$$
\tilde{z}= \begin{cases}1, & \text { in } \mathcal{N}(\partial \Omega, \delta) \\ 0, & \text { outside } \mathcal{N}(\partial \Omega, 2 \delta)\end{cases}
$$

We calculate

$$
\begin{gathered}
\int_{\mathcal{N}(\partial \Omega, \delta)} 1 d \mu_{t}^{\epsilon}<\int_{\mathcal{N}(\partial \Omega, \delta)} \tilde{z} d \mu_{t}{ }^{\epsilon}+\int_{\mathcal{N}(\partial \Omega, 2 \delta) \backslash \mathcal{N}(\partial \Omega, \delta)} \tilde{z} d \mu_{t}{ }^{\epsilon}+\int_{\Omega \backslash \mathcal{N}(\partial \Omega, 2 \delta)} \tilde{z} d \mu_{t}{ }^{\epsilon}=\int_{\Omega} \tilde{z} d \mu_{t}{ }^{\epsilon} \\
\Longleftrightarrow \int_{\mathcal{N}(\partial \Omega, \delta)} 1 d \mu_{t}{ }^{\epsilon}<\int_{\Omega} \tilde{z} d \mu_{t}{ }^{\epsilon}=\epsilon \sum_{j} \tilde{z}_{\xi_{j}}<\epsilon \cdot \operatorname{card}_{\mathcal{N}(\partial \Omega, 2 \delta)}\left(\xi_{j}\right)<\epsilon \cdot C \frac{\delta^{3}}{\epsilon}=C \delta^{3} \\
\Longleftrightarrow \int_{\mathcal{N}(\partial \Omega, \delta)} 1 d \mu_{t}<C \delta^{3}, \quad \text { by Lemma 3.2(v). }
\end{gathered}
$$

If we denote by $\lambda$ the Lebesgue measure, then (3.38) implies that $\mu_{t} \ll \lambda$ by utilizing

$$
\lambda(A)=\inf \left\{\sum_{n \in \mathbb{N}} \lambda\left(B_{n}\right): A \subset \bigcup_{n \in \mathbb{N}} B_{n}, B_{n} \text { balls }\right\} .
$$

As soon as $\mu_{t} \ll \lambda$, the Radon-Nikodym theorem provides us with the representation $\mu_{t}(A)=\int_{A} D_{\lambda} \mu_{t} d \lambda$ while it holds that $\left|D_{\lambda} \mu_{t}\right|=\left|d \mu_{t} / d \lambda\right|<C^{\prime} \lambda$ a.e. Now, it can be easily checked that (3.36) holds as soon as

$$
\left|\int_{\mathcal{N}(\partial \Omega, \delta)}\left(\frac{1}{|\xi-\eta|}+4 \pi \gamma(\xi, \eta)\right) \frac{d_{\mu_{t}}}{d \lambda} d \lambda\right| \leq \int_{\mathcal{N}(\partial \Omega, \delta)}\left(\frac{1}{|\xi-\eta|}+4 \pi \gamma(\xi, \eta)\right)\left|\frac{d_{\mu_{t}}}{d \lambda}\right| d \lambda \leq C^{\prime} \delta^{2}
$$

Hence, as $\delta, \epsilon \rightarrow 0,(3.31)$ is established.

The last step which will complete the proof is the estimation of the double sum $\epsilon(1 / N \bar{R}) \sum_{k, h, k \neq h}\left(R_{h} /\left|\xi_{h}-\xi_{k}\right|\right)\left(R_{k} / \bar{R}-1\right)$ and the determination of $K(t)$ in the limit. This will be accomplished in three steps.

Step 1. We show that

$$
\left|\epsilon \frac{1}{N \bar{R}} \sum_{\substack{k, h \\ k \neq h}} \frac{R_{h}}{\left|\xi_{h}-\xi_{k}\right|}\left(\frac{R_{k}}{\bar{R}}-1\right)+\epsilon \frac{1}{N} \sum_{i, h} 4 \pi \frac{R_{i}}{\bar{R}} \gamma\left(\xi_{i}, \xi_{h}\right)\left(\frac{R_{h}}{\bar{R}}-1\right)\right|<\mathscr{C}
$$


Indeed,

$$
\begin{aligned}
& \left|\epsilon \frac{1}{N \overline{\bar{R}}} \sum_{\substack{k, h \\
k \neq h}} \frac{R_{h}}{\left|\xi_{h}-\xi_{k}\right|}\left(\frac{R_{k}}{\bar{R}}-1\right)\right|+\left|\epsilon \frac{1}{N} \sum_{i, h} 4 \pi \frac{R_{i}}{\bar{R}} \gamma\left(\xi_{i}, \xi_{h}\right)\left(\frac{R_{h}}{\bar{R}}-1\right)\right| \\
& \quad \leq\left|\epsilon \frac{1}{N} \sum_{h} \frac{R_{h}}{\bar{R}} \sum_{k \neq h} \frac{1}{\left|\xi_{h}-\xi_{k}\right|}\left(\frac{R_{k}}{\bar{R}}-1\right)\right|+\left|\epsilon \frac{1}{N} 4 \pi \sum_{i} \frac{R_{i}}{\bar{R}} \sum_{h} \gamma\left(\xi_{i}, \xi_{h}\right)\left(\frac{R_{h}}{\bar{R}}-1\right)\right| \\
& \quad \leq \mathscr{C},
\end{aligned}
$$

utilizing the basic estimates (2.7), (2.14) for $\eta=1 / 3$ and Lemma 3.2(iii).

Step 2. As soon as the above double sum is bounded, there exists a sequence $\epsilon_{n}$ such that it converges to $K(t)$, uniformly in time.

Step 3. The constant $K(t)$ is determined via the conservation of volume formula to the limit so that the leading order reads:

$$
\frac{d}{d t} \int R^{3} d \mu_{t}=0
$$

Utilizing the definition of the measure, we have equivalently

$$
\begin{aligned}
& \frac{d}{d t}\left(\epsilon^{3 \eta} \sum_{i} R_{i}^{3}\right)=0 \Longleftrightarrow \epsilon^{3 \eta} \sum_{i} R_{i}^{2} \frac{d R_{i}}{d t}=0 \Longleftrightarrow \int R^{2} \frac{d R}{d t} d \mu_{t}=0 \\
& \Longleftrightarrow \int R^{2} \frac{1}{R}\left\{\left(\frac{1}{\bar{R}}-\frac{1}{R}\right)+K(t)-\int\left(\frac{1}{|\xi-\eta|}+4 \pi \gamma(\xi, \eta)\right)\left(\frac{R}{\bar{R}}-1\right) d \mu_{t}\right\} d \mu_{t}, \\
& \int\left(R K(t)-R \int\left(\frac{1}{|\xi-\eta|}+4 \pi \gamma(\xi, \eta)\right)\left(\frac{R}{\bar{R}}-1\right) d \mu_{t}\right) d \mu_{t}=0 \\
& \Longleftrightarrow K(t)=\frac{\iint R(1 /|\xi-\eta|+4 \pi \gamma(\xi, \eta))(R / \bar{R}-1) d \mu_{t}(R, \xi) d \mu_{t}(R, \eta)}{\int R d \mu_{t}} .
\end{aligned}
$$

As soon as the $\xi$ equations for $\eta=1 / 3$ tend to zero, the desired conservation law is an immediate consequence of (3.28) and Lemma 3.2(v).

Remark 3.4. Niethammer and Otto [8] obtained rigorous arguments based on homogenization for gradient flow structure under the hypotheses that the centers approximately do not move and the particles are radially symmetric. The case of the critical density is examined where the evolution for $\left\{R_{i}\right\}_{i}$ reduces only to an evolution of the joint distribution of particle radii and particle center $n(R, \xi, t)$. They derived the kinetic equation

$$
\partial_{t} n+\partial_{R}\left(\frac{d R}{d t} n\right)=0
$$


236 Continuum limits of particles interacting via diffusion

where

$$
\frac{d R}{d t}=\frac{1}{R^{2}}(R \bar{u}-1)
$$

and $\bar{u}$ satisfies the equation

$$
\Delta_{\xi} \bar{u}=4 \pi\left(\bar{u} \int R n d R-\int n d R\right)
$$

where $\bar{u}$ has now $\xi$ as a parameter contrary to the subcritical case where $\bar{u}$ is constant in space and satisfies $\bar{u}=\int n d R / \int R n d R$. We now show how this relates to (1.10):

$$
\frac{d R}{d t}=\frac{1}{R}\left\{\left(\frac{1}{\bar{R}}-\frac{1}{R}\right)+K(t)-\int_{0}^{\infty} \int_{\Omega}\left(\frac{1}{|\xi-\eta|}+4 \pi \gamma(\xi, \eta)\right)\left(\frac{R}{\bar{R}}-1\right) n(R, \eta, t) d \eta d R\right\}
$$

We introduce the length parameter $\lambda$ and set

$$
\tilde{u}=\frac{1}{\bar{R}}+\lambda K(t)-\lambda \int_{0}^{\infty} \int_{\Omega}\left(\frac{1}{|\xi-\eta|}+4 \pi \gamma(\xi, \eta)\right)\left(\frac{R}{\bar{R}}-1\right) n(R, \eta, t) d \eta d R
$$

We observe that our $\tilde{u}$ is the second approximation to the solution of (3.47). Indeed,

$$
\begin{aligned}
\Delta_{\xi} \tilde{u} & =-4 \pi \lambda \int_{0}^{\infty}\left(\frac{R}{\bar{R}}-1\right)\left[\Delta \xi \int_{\Omega}\left(\frac{1}{4 \pi|\xi-\eta|}+\gamma(\xi, \eta)\right) n(R, \eta, t) d \eta\right] d R \\
& =4 \pi \lambda \int_{0}^{\infty}\left(\frac{R}{\bar{R}}-1\right) n(R, \xi, t) d R \\
& =4 \pi \lambda\left[\frac{1}{\bar{R}} \int_{0}^{\infty} R n(R, \xi, t) d R-\int_{0}^{\infty} n(R, \xi, t) d R\right] \\
& =4 \pi \lambda\left[(\tilde{u}+O(\lambda)) \int_{0}^{\infty} R n(R, \xi, t) d R-\int_{0}^{\infty} n(R, \xi, t) d R\right] \\
& =4 \pi \lambda\left[\tilde{u} \int_{0}^{\infty} R n(R, \xi, t) d R-\int_{0}^{\infty} n(R, \xi, t) d R\right]+O\left(\lambda^{2}\right) .
\end{aligned}
$$

So, the desired result is obtained.

\section{Acknowledgments}

The first author acknowledges the hospitality and support of the Max Planck Institute for Mathematics in the Sciences, Leipzig, in the period from January to May 2002, during which this work was completed. The authors would also like to thank Professor P. W. Bates for several stimulating discussions. The third author was partially supported by a חENE $\Delta$ 99/527 interdisciplinary grant in materials. 


\section{References}

[1] N. D. Alikakos, P. W. Bates, and X. Chen, Convergence of the Cahn-Hilliard equation to the Hele-Shaw model, E Arch. Ration. Mech. Anal. 128 (1994), no. 2, 165-205.

[2] N. D. Alikakos and G. Fusco, The effect of distribution in space in Ostwald ripening, Nonlinear Dynamics and Renormalization Group (Montreal, QC, 1999), CRM Proc. Lecture Notes, vol. 27, American Mathematical Society, Rhode Island, 2001, pp. 17-28.

[3] _ Ostwald ripening for dilute systems under quasistationary dynamics, Comm. Math. Phys. 238 (2003), no. 3, 429-479.

[4] N. D. Alikakos, G. Fusco, and G. Karali, The effect of the geometry of the particle distribution in Ostwald ripening, Comm. Math. Phys. 238 (2003), no. 3, 481-488.

[5] D. Gilbarg and N. S. Trudinger, Elliptic Partial Differential Equations of Second Order, SpringerVerlag, Berlin, 1998.

[6] W. W. Mullins and R. F. Sekerka, Morphological stability of a particle growing by diffusion and heat flow, J. Appl. Physics 34 (1963), 323-329.

[7] B. Niethammer, Derivation of the LSW-theory for Ostwald ripening by homogenization methods, Arch. Ration. Mech. Anal. 147 (1999), no. 2, 119-178.

[8] B. Niethammer and F. Otto, Ostwald ripening: the screening length revisited, Calc. Var. Partial Differential Equations 13 (2001), no. 1, 33-68.

[9] B. Niethammer and J. J. Velázquez, Exponential screening and homogenization in coarsening systems, preprint, 2002.

[10] R. L. Pego, Front migration in the nonlinear Cahn-Hilliard equation, Proc. Roy. Soc. London Ser. A 422 (1989), no. 1863, 261-278.

[11] J. J. L. Velázquez, On the effect of stochastic fluctuations in the dynamics of the Lifshitz-SlyozovWagner model, J. Statist. Phys. 99 (2000), no. 1-2, 57-113.

[12] J. J. Weins and J. W. Cahn, The effects of size and distribution of second phase particles and voids on sintering, Sintering and Related Phenomena (G. C. Kuczynski, ed.), Plenum, New York, 1973, pp. 151-163.

Nicholas D. Alikakos: Department of Mathematics, University of Athens, 15784 Athens, Greece Current address: Department of Mathematics, University of North Texas, TX 76203, USA

E-mail address: nalikako@cc.uoa.gr

Giorgio Fusco: Dipartimento di Mathematica, Universita di L’Aquila, 67010 L’Aquila, Italy E-mail address: fusco@univaq.it

Georgia Karali: Department of Mathematics, University of Toronto, Toronto, Canada M5S 3G3 E-mail address: gkarali@math.toronto.edu 


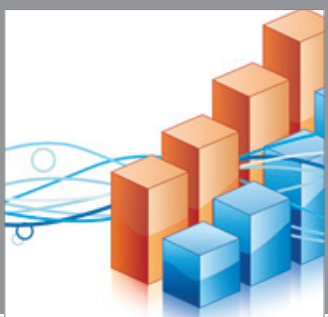

Advances in

Operations Research

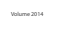

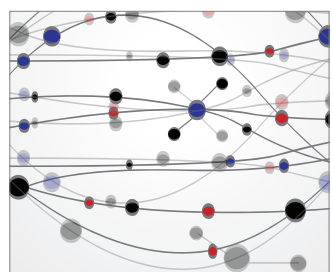

\section{The Scientific} World Journal
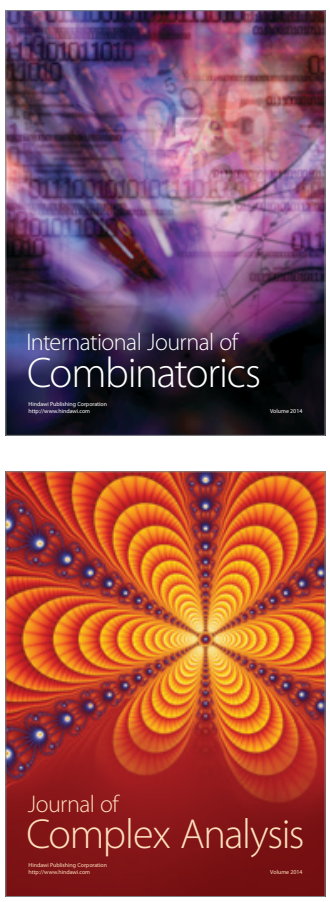

International Journal of

Mathematics and

Mathematical

Sciences
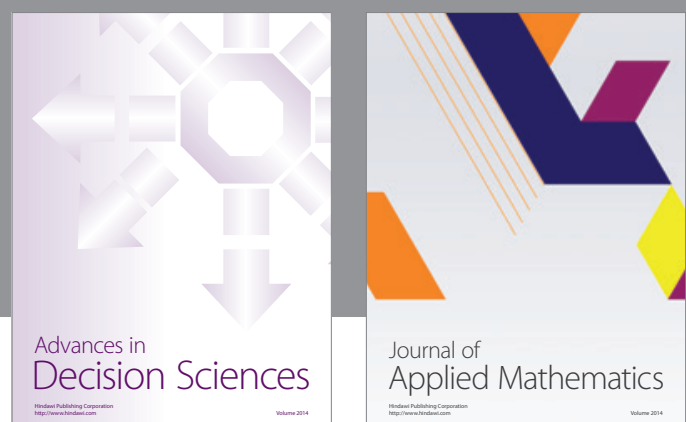

Journal of

Applied Mathematics
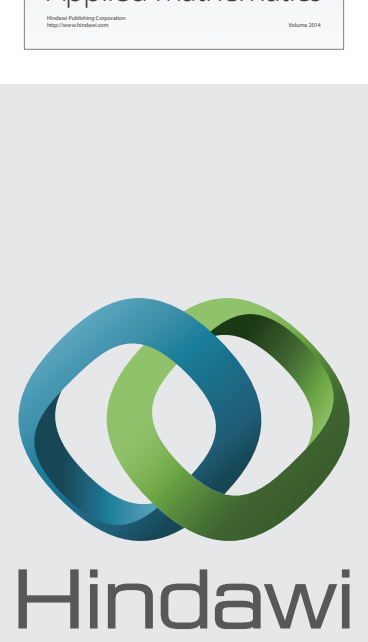

Submit your manuscripts at http://www.hindawi.com
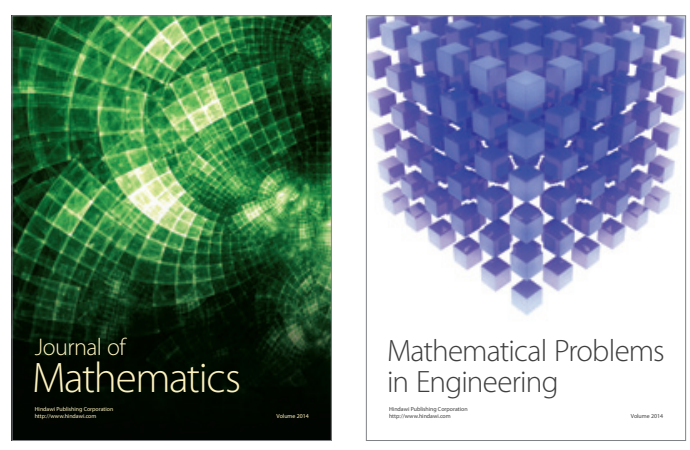

Mathematical Problems in Engineering
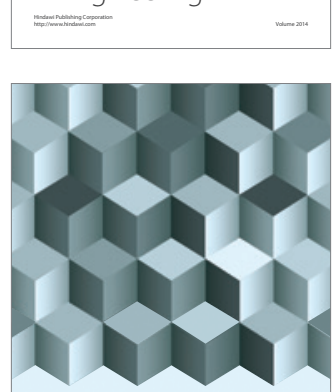

Journal of

Function Spaces
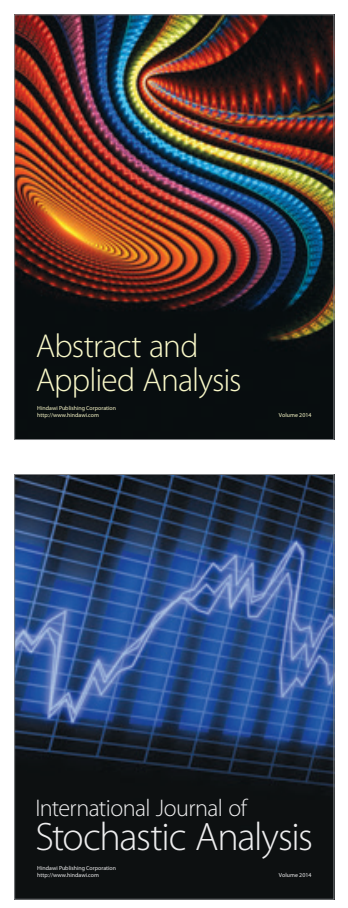

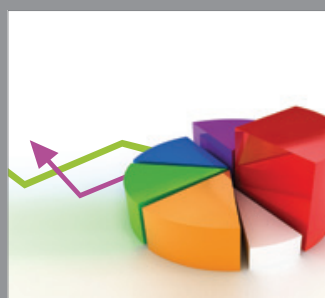

ournal of

Probability and Statistics

Promensencen
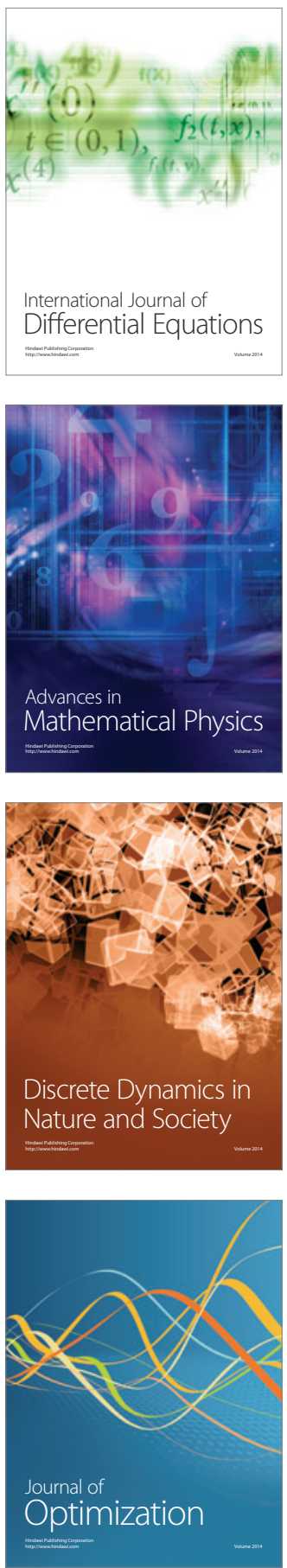